\title{
THE DARK SIDE OF SPORT: MANAGERIAL BULLYING AND HARASSMENT CHALLENGES IN DIFFERENT TYPES OF SPORTS
}

\author{
Jolita VVEINHARDT ${ }^{1}$, Vilija Bitė FOMINIENE $\dot{2}^{\star}$, Regina ANDRIUKAITIENE ${ }^{3}$ \\ ${ }^{1}$ Institute of Sport Science and Innovations, Lithuanian Sports University, Kaunas, Lithuania \\ ${ }^{2,}{ }^{3}$ Department of Sport and Tourism management, Lithuanian Sports University, \\ Kaunas, Lithuania
}

Received 10 February 2020; accepted 05 May 2020

\begin{abstract}
Bullying and harassment are the dark side of sport, not open to spectators and often ignored by coaches and leaders. Therefore, the research aim is to identify prevalence of bullying and harassment in Lithuanian organized sport with regard to individual, combat and team sports, highlighting measures/actions used to manage these phenomena. Survey research uses quantitative research strategy. The study involved 1440 organized sports athletes representing all sports types. The prevalence of bullying actions with regard to separate type of sports was identified: in individual sports $(9.8 \%)$, in combat sports $(8.5 \%)$, and in team sports ( $7.3 \%)$. The analysis by four specific actions has shown that athletes experienced most of such actions in combat sports (20\%); almost half less, in team sports (10.8\%) and individual sports (10.1\%). The results of the study show a lack of bullying and harassment intervention and of comprehensive complaint submission procedures, while coaches particularly often ignore athletes' negative behaviour. The article discusses managerial causes of bullying and harassment in the sports organization, presenting a holistic view of the phenomenon, including the forms of violence that have been little studied so far. This will help leaders to carry out prevention and intervention activities the specificity of which comes to prominence depending on the types of sports.
\end{abstract}

Keywords: bullying, harassment, sport organization, types of sports, management, intervention, BHISQ, Lithuania.

JEL Classification: L83, M19, I30, Z29.

\section{Introduction}

Sport performance as a product is offered to buyers and primarily to its all participants. The demand for such participant product is growing in the society alongside with the approach that sport participation, especially in organized sports, is becoming one of the main forms of physical activity for youth (Holt et al., 2017). But only when the environment of such activities is protective and nurturing, the creation of interpersonal relationships in sport

*Corresponding author. E-mail: vilija.fominiene@lsu.lt

Copyright (c) 2020 The Author(s). Published by Vilnius Tech Press

This is an Open Access article distributed under the terms of the Creative Commons Attribution License (http://creativecommons. org/licenses/by/4.0/), which permits unrestricted use, distribution, and reproduction in any medium, provided the original author and source are credited. 
performance can become potential determinants of a positive athlete's development (Holt et al., 2017) and lead to positive physical and mental health and social well-being. Naturally, this becomes a managerial challenge for various sports organizations implementing sport performance based on organized sport (Kerr et al., 2013; Donohue et al., 2015). And on a particularly pressing issue discussing amateur sport, as the pursuit of results and victories encourages those persons who are involved in governing these activities to act contrary to the fundamentals of sports and especially with regard to young people. In this case, both the professional behaviour of the organization's management activities and sports managers (Škorić, 2018) and the implementation of some coaching tactics (Merkel, 2013) contributed not to the positive but to the negative atmosphere in sports and consequences for participants. Still, one of the responsibilities of the sport organization is the welfare of the athletes; i.e., those who are named in the Sport Industry Segment Model as buyers of a participant product (Pitts et al., 1994).

In recent years, analysing factors determining negative atmosphere as part of sports environment, such as antisocial behaviour, aggression, violence and abuse, sports participants' bullying and harassment behaviour is distinguished too. In summary, such behaviour falls under the umbrella of athlete relational maltreatment (Stirling \& Kerr, 2016) and is treated as repeated and persistent negative behaviour (according to Notelaers et al., 2019). There is no lack of research in scientific literature analysing aggression, violence or abuse in sport at both horizontal, peer-to-peer and vertical levels, but it is acknowledged that bullying and harassment are a complex challenge encountered by most of sports organization leaders and, unfortunately, they are still being poorly studied (Evans et al., 2016; Fisher \& Dzikus, 2017). However, the increased requirements for a safe environment in which sports take place in recent years have also determined the number of studies analysing bullying and harassment behaviour in sport (Yildiz, 2015; Evans et al., 2016; Kerr et al., 2016; Peltola \& Kivijärvi, 2017; Jewett et al., 2019; Nery et al., 2019; Mishna et al., 2019). In recent years the number of such studies has increased in Lithuania as well (Vveinhardt et al., 2017, 2019; Vveinhardt $\&$ Fominiene, 2020a). These studies, although conducted using various instruments and employing different methodological approaches or interpreted using different concepts of the phenomenon, only accentuate the problem of bullying and harassment in sport and the need for effective prevention (Vveinhardt et al., 2017) and extensive intervention that is often still insufficient (Nery et al., 2019). In addition, due to insufficient awareness of the phenomenon, managers of sports activities and organizations fail to develop appropriate bullying response protocols (Shannon, 2013), and organizational tolerance promotes escalation of violent actions (Roberts et al., 2019).

The research aim is to identify the prevalence of bullying and harassment in Lithuanian organized sport with regard to individual, combat and team sports types, highlighting measures/actions for management of these phenomena. Three problem questions are formulated to this end: What is the prevalence of bullying and harassment in sports organizations by frequency and nature of malicious actions; Which bullying and harassment management measures are used in teams and groups of athletes; What intervention actions at the organizational level, distinguished by athletes, help to cope with bullying and which individuals provided support. 
In order to achieve the aim of the research the paper is organized as follows: section 1 provides an overview of the literature regarding bullying and harassment phenomena and its expression in sports; section 2 presents research approaches and explains detailed structure of the questionnaire; section 3 presents data and results, section 4 discusses the findings and last section provides the conclusions.

\section{Literature review}

Bullying and harassment constitute serious and complex social problems, which received widespread attention over the years. The first systematic description of the bullying phenomenon was provided in 1972 by Heinemann, who investigated the nature of bullying at school. The term harassment was used in 1976 in the US by Brodsky, focusing on the harassed worker. During the mid-1990s, researchers from European countries, especially Scandinavia, began to extensively analyse bullying and harassment in the workplace (see in Gillen et al., 2004). This phenomenon was also widely analysed investigating the educational context (Smith, 2016; Menesini \& Salmivalli, 2017). It is likely that the abundance of these studies has also partly determined the view that bullying is characteristic to childhood and adolescence (Lund \& Ross, 2017; Bachand, 2017). However, it is evident that this negative aspect of interpersonal relationships also manifests itself in other social contexts, regardless of participants' age, gender or other characteristics, and causes various harmful effects (Twemlow \& Sacco, 2013). Studies on the consequences of bullying and harassment mostly identify problems of physical, mental and social health, which a person may experience, having found himself/herself in bullying situations. Moore et al. (2017), who have performed a systematic review and meta-analysis, found that bullying victimization could determine various negative mental health outcomes such as poor mental health, syndromes of depression and anxiety, and psychotic symptoms, suicidal ideation and suicide attempts. Besides, bullying victimization associates with the increased risk of somatic symptoms encompassing such distinguished symptoms as stomach ache, sleeping difficulties, headaches, dizziness, and back pain. Poor life satisfaction or a sense of loneliness are also noted as well as problems related to alcohol or drug use.

Considering potentially serious and long-term consequences of this phenomenon and looking for effective anti-bullying programs, it becomes very important to clearly name this phenomenon and define circumstances under which it occurs. Unfortunately, the growing interest in this phenomenon not only increases the number of conducted studies in different contexts but also increasingly clearly reveals the prevailing problem of terms and definitions, raised by Crawshaw (2009) as early as in 2009.

Nowadays, it is widely accepted that bullying and harassment have become part of social life and can be described as a specific type of relational aggression. However, in the scientific literature, the term bullying is used slightly more often. In the Western research tradition, it means intentional, repetitive aggressive behaviours, including some sort of power imbalance against a specific target (victim) that cannot defended himself/herself easily, and this is treated as a systematic abuse of power (see in Smith et al., 2013; Hellström et al., 2015). Despite this, analysing this negative phenomenon, it is accompanied by the term harassment 
(Notelaers \& Van der Heijden, 2019), although there are researchers stating that harassment can sometimes differ from bullying in that it can only occur as a single event and not as repeated behaviour directed towards another person, while differences in the status of the victim and aggressor do not necessarily exist (Cosgrove \& Nickerson, 2017). In this case, the terms bullying and harassment can be used both interchangeably and collectively, and can be defined as "unwanted aggressive behaviours" that "inflict harm or distress", and are "repeated multiple times or are highly likely to be repeated" in the context of an "observed or perceived power differential" (Patchin \& Hinduja, 2015). It is also appropriate to use such conception in the context of sport, investigating bullying and harassment manifestations.

Analysing this phenomenon in sport, attention is also drawn to the fact that bullying and harassment are grounded on the power imbalance between two/some individuals. This means that in organized sports activities, both in team and individual sports, such negative situations can be experienced by any participant of the sports activity: coach-youth athlete, coach-adult athlete, youth athlete-youth athlete, adult athlete-adult athlete, adult athleteyouth athlete (Stirling et al., 2011; Bachand, 2017). Studies have found that this is the case really, while the encounter with the acts of harm also causes various negative consequences for both the sports participant and sports organization (Fisher \& Dzikus, 2017). This can be prevented only by efficiently applied preventive measures and effective intervention programs grounded on research results. However, the currently existing system in the sport sector is insufficiently effective, although it provides for youth sports guidelines, rules and regulations (Shannon, 2013). First, this pertains to the system of penalties, grounded on zero tolerance. Although there is no convincing foundation that punishments deter misconduct, they are often used and include such measures as temporary exclusion from the competition or training or the athlete's expulsion from the sport organisation itself (McMullen, 2014). Second, it is recognised that this system lacks scientific substantiation of incidence, manifestation and nature of various negative phenomena (Merkel, 2013). Consequently, sports organisations still do not have clear and effective anti-bullying policies that could be stated at all levels of the organisation. Often it is unclear how to inform about the cases of bullying and harassment (Stefaniuk \& Bridel, 2018).

\section{Research methodology}

Measures. The Bullying and harassment in sport questionnaire (BHISQ) was used to conduct the survey. The detailed structure of the questionnaire is presented in Table 1.

The research results section presents that part which is exclusively related only to bullying and harassment actions in sport, lack of bullying and harassment control/management, trustworthy persons and actions that have helped to endure bullying and harassment (Table 1: sections II, IV and VI).

Participants. The survey involved 1440 participants of organized sports, of these 1011 male and 439 female representatives. Respondents represented all three sports types; i.e., individual sports $(\mathrm{N}=134$ males and $\mathrm{N}=209$ females $)$, combat sports $(\mathrm{N}=39$ males and $\mathrm{N}=20$ females $)$ and team sports $(\mathrm{N}=828$ males and $\mathrm{N}=210$ females $)$, and participated in amateur sports activities at least 6 months. 
Table 1. Structure of the Bullying and harassment in sport questionnaire (BHISQ) (source: Vveinhardt \& Fominienè, 2020b)

\begin{tabular}{|c|c|c|c|c|c|c|}
\hline \multicolumn{2}{|c|}{ Parts } & \multicolumn{2}{|l|}{ Scales } & \multicolumn{2}{|l|}{ Subscales } & \multirow{2}{*}{$\begin{array}{c}\mathrm{N} \text { of } \\
\text { items }\end{array}$} \\
\hline \multirow{7}{*}{$\begin{array}{l}\text { I. Anti-social } \\
\text { behaviour } \\
\text { and } \\
\text { aggression in } \\
\text { sport }\end{array}$} & \multirow{7}{*}{ ASBAS } & \multirow{2}{*}{$\begin{array}{l}\text { 1.1. Antisocial } \\
\text { behaviour in sport }\end{array}$} & \multirow{2}{*}{ ABS } & $\begin{array}{l}\text { 1.1.1. Antisocial } \\
\text { teammate }\end{array}$ & AT & \\
\hline & & & & $\begin{array}{l}\text { 1.1.2. Antisocial } \\
\text { opponent }\end{array}$ & $\mathrm{AO}$ & $8^{1}$ \\
\hline & & \multirow{2}{*}{$\begin{array}{l}\text { 1.2. Aggression and } \\
\text { anger in competition }\end{array}$} & \multirow{2}{*}{$\mathrm{AAC}$} & 1.2.1. Anger & AN & $6^{2}$ \\
\hline & & & & 1.2.2. Aggressiveness & AG & $6^{2}$ \\
\hline & & \multirow{3}{*}{$\begin{array}{l}\text { 1.3. Types of } \\
\text { aggression during } \\
\text { trainings and } \\
\text { competitions }\end{array}$} & \multirow{3}{*}{ TATC } & $\begin{array}{l}\text { 1.3.1. Physical } \\
\text { aggression }\end{array}$ & PA & $7^{3^{*}}$ \\
\hline & & & & $\begin{array}{l}\text { 1.3.2. Verbal } \\
\text { aggression }\end{array}$ & VA & $8^{3^{*}}$ \\
\hline & & & & $\begin{array}{l}\text { 1.3.3. Indirect } \\
\text { aggression }\end{array}$ & IA & $8^{3^{*}}$ \\
\hline \multirow{9}{*}{$\begin{array}{l}\text { II. Bullying } \\
\text { and } \\
\text { harassment } \\
\text { actions in } \\
\text { sport }\end{array}$} & \multirow{9}{*}{ BHAIS } & \multirow{2}{*}{$\begin{array}{l}\text { 2.1. Athletes, } \\
\text { initiating and } \\
\text { experiencing } \\
\text { bullying }\end{array}$} & \multirow[b]{2}{*}{ AIEB } & 2.1.1. Bully role & $\mathrm{BR}$ & $9^{4}$ \\
\hline & & & & 2.1.2. Victim role & VR & $4^{4}$ \\
\hline & & \multirow{2}{*}{$\begin{array}{l}\text { 2.2. Commu- } \\
\text { nication obstacles } \\
\text { in teammates inter- } \\
\text { relationship }\end{array}$} & \multirow[b]{2}{*}{ COTI } & 2.2.1. Communication & $\mathrm{CO}$ & $10^{5}$ \\
\hline & & & & 2.2.2. Isolation & IS & $6^{5}$ \\
\hline & & \multirow{2}{*}{$\begin{array}{l}\text { 2.3. Formation of } \\
\text { negative approach } \\
\text { and the pattern of } \\
\text { sport tasks }\end{array}$} & \multirow[b]{2}{*}{ FNAT } & 2.3.1. Reputation & $\mathrm{RE}$ & $17^{5^{*}}$ \\
\hline & & & & 2.3.2. Tasks & TA & $9^{5^{*}}$ \\
\hline & & \multirow{3}{*}{$\begin{array}{l}\text { 2.4. Teammates' } \\
\text { feelings and } \\
\text { consequences }\end{array}$} & \multirow{3}{*}{ TFC } & 2.4.1. Health & $\mathrm{HE}$ & $6^{5^{*}}$ \\
\hline & & & & 2.4.2. Damage & DA & $5^{5^{*}}$ \\
\hline & & & & 2.4.3. Frustration & FR & $6^{*}$ \\
\hline \multirow{4}{*}{$\begin{array}{l}\text { III. Previous } \\
\text { bullying and } \\
\text { harassment } \\
\text { experiences } \\
\text { and } \\
\text { memories }\end{array}$} & \multirow{4}{*}{ PBHEM } & \multirow{2}{*}{$\begin{array}{l}\text { 3.1. Bullying } \\
\text { and harassment } \\
\text { experience }\end{array}$} & \multirow[b]{2}{*}{ BHE } & $\begin{array}{l}\text { 3.1.1. Experience at } \\
\text { school }\end{array}$ & ES & $5^{7}$ \\
\hline & & & & $\begin{array}{l}\text { 3.1.2. Experience in } \\
\text { the previous team/ } \\
\text { group }\end{array}$ & ET & $5^{7^{*}}$ \\
\hline & & \multirow{2}{*}{$\begin{array}{l}\text { 3.2. Bullying } \\
\text { and harassment } \\
\text { memories }\end{array}$} & \multirow{2}{*}{ BHM } & $\begin{array}{l}\text { 3.2.1. School } \\
\text { memories }\end{array}$ & SM & $5^{7}$ \\
\hline & & & & $\begin{array}{l}\text { 3.2.2. Memories from } \\
\text { a previous team/group }\end{array}$ & $\mathrm{TM}$ & $5^{7^{*}}$ \\
\hline \multirow{3}{*}{$\begin{array}{l}\text { IV. Lack of } \\
\text { bullying and } \\
\text { harassment } \\
\text { control }\end{array}$} & \multirow{3}{*}{ LBHCO } & \multirow{3}{*}{$\begin{array}{l}\text { 4.1. Affirmation, } \\
\text { intervention } \\
\text { and prevention } \\
\text { of bullying and } \\
\text { harassment }\end{array}$} & \multirow{3}{*}{ IPBH } & $\begin{array}{l}\text { 4.1.1. Seeing the } \\
\text { situation, fact } \\
\text { statement }\end{array}$ & FS & $4^{* *}$ \\
\hline & & & & $\begin{array}{l}\text { 4.1.2. Bullying } \\
\text { intervention }\end{array}$ & BI & $8^{* *}$ \\
\hline & & & & $\begin{array}{l}\text { 4.1.3. Bullying } \\
\text { prevention }\end{array}$ & $\mathrm{BP}$ & $4^{* *}$ \\
\hline
\end{tabular}


End of Table 1

\begin{tabular}{|c|c|c|c|c|c|c|}
\hline \multicolumn{2}{|c|}{ Parts } & \multicolumn{2}{|l|}{ Scales } & \multicolumn{2}{|l|}{ Subscales } & $\begin{array}{l}\mathrm{N} \text { of } \\
\text { items }\end{array}$ \\
\hline \multirow{3}{*}{$\begin{array}{l}\text { V. Bullying } \\
\text { and } \\
\text { harassment } \\
\text { participants }\end{array}$} & \multirow{3}{*}{ BAHPA } & \multirow{3}{*}{$\begin{array}{l}5.1 \text {. Victim, } \\
\text { bystander and } \\
\text { bully among one's } \\
\text { own and against } \\
\text { opponents }\end{array}$} & \multirow{3}{*}{ VIBB } & 5.1.1. Victim & VI & $2^{6}$ \\
\hline & & & & 5.1.2. Bystander & BY & $2^{6}$ \\
\hline & & & & 5.1.3. Bully & $\mathrm{BU}$ & $2^{6}$ \\
\hline \multirow{4}{*}{$\begin{array}{l}\text { VI. } \\
\text { Trustworthy } \\
\text { persons and } \\
\text { actions that } \\
\text { have helped } \\
\text { to endure } \\
\text { bullying }\end{array}$} & \multirow{4}{*}{ ТРАНВ } & \multirow{2}{*}{$\begin{array}{l}\text { 6.1. Trustworthy } \\
\text { persons from the } \\
\text { victim's standpoint }\end{array}$} & \multirow[t]{2}{*}{ TPVS } & $\begin{array}{l}\text { 6.1.1. Less close } \\
\text { persons and/or } \\
\text { strangers } \\
\text { who can help }\end{array}$ & LP & $9^{7^{*}}$ \\
\hline & & & & $\begin{array}{l}\text { 6.1.2. Persons in } \\
\text { the immediate } \\
\text { environment }\end{array}$ & PI & $6^{7^{*}}$ \\
\hline & & \multirow{2}{*}{$\begin{array}{l}\text { 6.2. Actions that } \\
\text { have helped to } \\
\text { endure bullying }\end{array}$} & \multirow[b]{2}{*}{ AHEB } & $\begin{array}{l}\text { 6.2.1. Informing and } \\
\text { conversations }\end{array}$ & IC & $4^{7^{*}}$ \\
\hline & & & & $\begin{array}{l}\text { 6.2.2. Preventive } \\
\text { education and } \\
\text { self-development }\end{array}$ & ED & $7^{7^{*}}$ \\
\hline
\end{tabular}

Notes:

${ }^{1} 12$ items (I part) - "Prosocial and Antisocial Behaviour in Sport Scale" (PABSS) (Kavussanu \& Boardley, 2009).

${ }^{2} 12$ items (I part) - "Competitive Aggressiveness and Anger Scale" (CAAS) (Maxwell \& Moores, 2007).

${ }^{3} 23$ items (I part) - "Buss-Durkee Hostility Inventory" (BDHI) (Buss \& Durkee, 1957).

${ }^{4} 13$ items (II part) - "Illinois Bully Scale" (IBS) (Espelage \& Holt, 2001).

${ }^{5} 53$ items (II part) - Shortened version of "Mobbing as discrimination in employee relations aiming to improve the organisational climate" (MDOC-110) (Vveinhardt, 2012), i.e. "Mobbing and Single Cases of Harassment in Employees' Relations" (MSCH-47) (Vveinhardt \& Streimikiene, 2015), "Bullying and harassment in junior/youth team sport-40" (Vveinhardt et al., 2017).

${ }^{6} 6$ items (V part) - double-check questions, i.e. control questions.

746 items, i.e. 20 items (III part), 26 items (VI part) - "Bullying and Single Cases of Harassment in Higher Education Institutions" (B-SCH-St) (Vveinhardt et al., 2020) and "Bullying and Single Cases of Harassment in Professional Sport Teams" (B-SCH-Sp) (Vveinhardt \& Andriukaitienè, 2017).

* combined and adapted items.

** 16 items (IV part) - based on the analysis of scientific literature.

The probability sampling technique - random sampling was chosen for the study. From a statistical point of view, this sampling gives every member of the population an equal chance/ probability of being included. The number of surveyed subjects and distribution by variables according to the data of Lithuanian sports statistics of 2017 represent the whole population of young athletes in Lithuania. 
Procedure. The study was carried out in 2019. As it was intended to conduct the study with the youth population (respondents' age was from 16 to 29), the permission of the Ethics Supervision Committee of Research in Social Sciences of Lithuanian Sports University was received (No. SMTEK-3). Considering the sensitivity of some of the questions given in the questionnaire, in the request to issue a permit to conduct a social study, the authors of this research committed to depersonalize the questionnaires, ensuring the respondents' anonymity and confidentiality, and to store the collected survey data in personal archives without transferring them to third parties.

The paper and online questionnaires for data collection were chosen. To obtain permission to carry out a survey the researches applied to top managers of sports organizations clubs or sports teams and team's coaches, informing them about the study objectives and procedures. With their consent, the athletes were asked to complete the selected questionnaires. Prior to this, all participants were given verbal instructions on the study and their anonymity, confidentiality and voluntary nature were assured.

Data Analysis. Mean values, standard deviations and percentage distribution of the responses were calculated. Differences between groups were estimated using one-factor dispersion analysis (ANOVA). Chi-squared test was applied for testing relationships between categorical variables. Differences were considered statistically significant when values approached $\mathrm{p}<.05$ level. Statistical analysis of the research data was carried out using SPSS (Statistical Package of the Social Science) version 24.0.

\section{Research results}

The frequency of bullying and harassment actions is measured from "daily to once per week" (which corresponds to the definition of bullying) to "at least once per month to once every few months" (which indicates isolated incidents of bullying). The nature of bullying and harassment actions in athlete interrelationships is analysed at the subscale level and further elaborated by respective actions (not only): found fault, ridiculed, called names, struck (Table 2).

Chi-square test results show that in many cases, bullying and harassment actions and their frequency significantly vary depending on the type of sports to which the athletes who have experienced bullying and harassment are attributed. The results presented separately by types of sports showed that the highest percentages of bullying prevalence in sport (the mean is derived from seven subscales of bullying actions; i.e., communication; isolation; reputation; tasks; health; damage; frustration), were in individual (9.8\%), combat (8.5\%), and team sports types $(7.3 \%)$.

Analysing four specific actions separately (found faults, ridiculed, called names, struckdaily or once a week), again, having derived the mean, it was found that athletes experienced most of such actions in combat sports (20\%); almost half less, in team sports (10.8\%) and individual sports groups (10.1\%) (Table 3). This shows that the situation in different sports groups requires specific approach, putting greater focus on the "hottest" areas of risk-causing malicious actions. 
1532 J. Vveinhardt et al. The dark side of sport: managerial bullying and harassment challenges in different...

Table 2. Frequency and nature of bullying and harassment actions: individual, combat, team sports types (a)

\begin{tabular}{|c|c|c|c|c|c|c|c|c|c|c|c|}
\hline \multirow{2}{*}{\multicolumn{4}{|c|}{$\begin{array}{l}\text { Bullying and harassment } \\
\text { actions in sport }\end{array}$}} & \multirow{2}{*}{\multicolumn{2}{|c|}{$\begin{array}{c}\text { Individual } \\
\text { sports } \mathrm{N}=343\end{array}$}} & \multirow{2}{*}{\multicolumn{2}{|c|}{$\begin{array}{l}\text { Combat sports } \\
\mathrm{N}=59 \\
\text { Quantity } \mid \%\end{array}$}} & \multirow{2}{*}{\multicolumn{2}{|c|}{$\begin{array}{l}\text { Team sports } \\
\mathrm{N}=1038 \\
\text { Quantity } \mid \%\end{array}$}} & \multicolumn{2}{|c|}{$\begin{array}{l}\text { Chi-square test } \\
\text { results }\end{array}$} \\
\hline & & & & & & & & & & \multirow{2}{*}{$\frac{\chi^{2}}{18.733}$} & \multirow{2}{*}{$\frac{\mathrm{P}}{0.0001}$} \\
\hline \multirow{8}{*}{\multicolumn{2}{|c|}{ 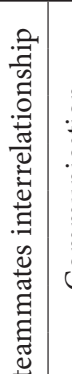 }} & \multirow{2}{*}{$\begin{array}{l}\text { Once every } \\
\text { few months }\end{array}$} & DNE & 216 & 63.0 & 31 & 52.5 & 514 & 49.5 & & \\
\hline & & & EXP & 127 & 37.0 & 28 & 47.5 & 524 & 50.5 & & \\
\hline & & \multirow{2}{*}{$\begin{array}{l}\text { At least once } \\
\text { per month }\end{array}$} & DNE & 279 & 81.3 & 47 & 79.7 & 822 & 79.2 & 0.738 & 0.692 \\
\hline & & & EXP & 64 & 18.7 & 12 & 20.3 & 216 & 20.8 & & \\
\hline & & \multirow{2}{*}{$\begin{array}{l}\text { Once per } \\
\text { week }\end{array}$} & DNE & 312 & 91.0 & 53 & 89.8 & 950 & 91.5 & 0.274 & 0.872 \\
\hline & & & EXP & 31 & 9.0 & 6 & 10.2 & 88 & 8.5 & & \\
\hline & & \multirow{2}{*}{ Daily } & DNE & 325 & 94.8 & 55 & 93.2 & 1002 & 96.5 & 3.317 & 0.190 \\
\hline & 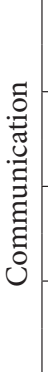 & & EXP & 18 & 5.2 & 4 & 6.8 & 36 & 3.5 & & \\
\hline \multirow{8}{*}{ 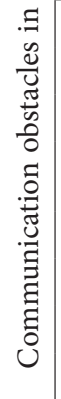 } & \multirow{8}{*}{ 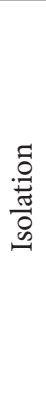 } & \multirow{2}{*}{$\begin{array}{l}\text { Once every } \\
\text { few months }\end{array}$} & DNE & 284 & 82.8 & 45 & 76.3 & 813 & 78.3 & 3.491 & 0.175 \\
\hline & & & EXP & 59 & 17.2 & 14 & 23.7 & 225 & 21.7 & & \\
\hline & & \multirow{2}{*}{\begin{tabular}{|l|} 
At least \\
once \\
per month
\end{tabular}} & DNE & 314 & 91.5 & 53 & 89.8 & 963 & 92.8 & 1.111 & 0.574 \\
\hline & & & EXP & 29 & 8.5 & 6 & 10.2 & 75 & 7.2 & & \\
\hline & & \multirow{2}{*}{$\begin{array}{l}\text { Once per } \\
\text { week }\end{array}$} & DNE & 328 & 95.6 & 54 & 91.5 & 1007 & 97.0 & 5.833 & 0.048 \\
\hline & & & EXP & 15 & 4.4 & 5 & 8.5 & 31 & 3.0 & & \\
\hline & & \multirow{2}{*}{ Daily } & DNE & 331 & 96.5 & 58 & 98.3 & 1023 & 98.6 & 5.722 & 0.050 \\
\hline & & & EXP & 12 & 3.5 & 1 & 1.7 & 15 & 1.4 & & \\
\hline \multirow{8}{*}{ 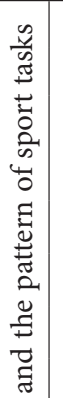 } & \multirow{8}{*}{ 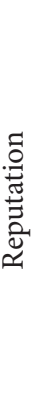 } & \multirow{2}{*}{$\begin{array}{l}\text { Once every } \\
\text { few months }\end{array}$} & DNE & 228 & 66.5 & 34 & 57.6 & 551 & 53.1 & 18.836 & 0.0001 \\
\hline & & & EXP & 115 & 33.5 & 25 & 42.4 & 487 & 46.9 & & \\
\hline & & \multirow{2}{*}{$\begin{array}{l}\text { At least once } \\
\text { per month }\end{array}$} & DNE & 301 & 87.8 & 48 & 81.4 & 804 & 77.5 & 17.198 & 0.001 \\
\hline & & & EXP & 42 & 12.2 & 11 & 18.6 & 234 & 22.5 & & \\
\hline & & \multirow{2}{*}{$\begin{array}{l}\text { Once per } \\
\text { week }\end{array}$} & DNE & 318 & 92.7 & 56 & 94.9 & 963 & 92.8 & 0.398 & 0.820 \\
\hline & & & EXP & 25 & 7.3 & 3 & 5.1 & 75 & 7.2 & & \\
\hline & & \multirow{2}{*}{ Daily } & DNE & 321 & 93.6 & 57 & 96.6 & 1020 & 98.3 & 19.989 & 0.0001 \\
\hline & & & EXP & 22 & 6.4 & 2 & 3.4 & 18 & 1.7 & & \\
\hline ت्t & \multirow{8}{*}{$\frac{\mathscr{y}}{\tilde{y}}$} & & DNE & 273 & 79.6 & 41 & 69.5 & 705 & 67.9 & 17.027 & 0.0001 \\
\hline : & & few months & EXP & 70 & 20.4 & 18 & 30.5 & 333 & 32.1 & & \\
\hline בั & & At least once & DNE & 316 & 92.1 & 52 & 88.1 & 857 & 82.6 & 19.028 & 0.0001 \\
\hline ه్ర & & & EXP & 27 & 7.9 & 7 & 11.9 & 181 & 17.4 & & \\
\hline प्र & & Once per & DNE & 322 & 93.9 & 54 & 91.5 & 962 & 92.7 & 0.744 & 0.689 \\
\hline .0. & & & EXP & 21 & 6.1 & 5 & 8.5 & 76 & 7.3 & & \\
\hline$\Xi$ & & Drily & DNE & 324 & 94.5 & 58 & 98.3 & 1002 & 96.5 & 3.751 & 0.153 \\
\hline I & & & EXP & 19 & 5.5 & 1 & 1.7 & 36 & 3.5 & & \\
\hline
\end{tabular}


End of Table 2

\begin{tabular}{|c|c|c|c|c|c|c|c|c|c|c|c|}
\hline \multirow{2}{*}{\multicolumn{4}{|c|}{$\begin{array}{l}\text { Bullying and harassment } \\
\text { actions in sport }\end{array}$}} & \multirow{2}{*}{\multicolumn{2}{|c|}{$\begin{array}{c}\begin{array}{c}\text { Individual } \\
\text { sports } \mathrm{N}=343\end{array} \\
\text { Quantity } \mid \%\end{array}$}} & \multirow{2}{*}{\multicolumn{2}{|c|}{$\begin{array}{l}\text { Combat sports } \\
\qquad \mathrm{N}=59 \\
\text { Quantity } \mid \%\end{array}$}} & \multirow{2}{*}{\multicolumn{2}{|c|}{$\begin{array}{l}\text { Team sports } \\
\mathrm{N}=1038 \\
\text { Quantity } \mid \%\end{array}$}} & \multicolumn{2}{|c|}{$\begin{array}{l}\text { Chi-square test } \\
\text { results }\end{array}$} \\
\hline & & & & & & & & & & \multirow{2}{*}{$\frac{\chi^{2}}{0.706}$} & \multirow{2}{*}{$\frac{P}{0.703}$} \\
\hline \multirow{24}{*}{$\begin{array}{l}\tilde{u} \\
\tilde{\Xi} \\
0\end{array}$} & \multirow{8}{*}{ 声 } & \multirow{2}{*}{$\begin{array}{l}\text { Once every } \\
\text { few months }\end{array}$} & DNE & 283 & 82.5 & 46 & 78.0 & 851 & 82.0 & & \\
\hline & & & EXP & 60 & 17.5 & 13 & 22.0 & 187 & 18.0 & & \\
\hline & & \multirow{2}{*}{$\begin{array}{l}\text { At least once } \\
\text { per month }\end{array}$} & DNE & 319 & 93.0 & 57 & 96.6 & 980 & 94.4 & 1.601 & 0.449 \\
\hline & & & EXP & 24 & 7.0 & 2 & 3.4 & 58 & 5.6 & & \\
\hline & & \multirow{2}{*}{$\begin{array}{l}\text { Once per } \\
\text { week }\end{array}$} & DNE & 324 & 94.5 & 57 & 96.6 & 1012 & 97.5 & 7.522 & 0.023 \\
\hline & & & EXP & 19 & 5.5 & 2 & 3.4 & 26 & 2.5 & & \\
\hline & & \multirow{2}{*}{ Daily } & DNE & 333 & 97.1 & 58 & 98.3 & 1016 & 97.9 & 0.827 & 0.661 \\
\hline & & & EXP & 10 & 2.9 & 1 & 1.7 & 22 & 2.1 & & \\
\hline & \multirow{8}{*}{ ค̊ } & \multirow{2}{*}{$\begin{array}{l}\text { Once every } \\
\text { few months }\end{array}$} & DNE & 319 & 93.0 & 53 & 89.8 & 961 & 92.6 & 0.737 & 0.692 \\
\hline & & & EXP & 24 & 7.0 & 6 & 10.2 & 77 & 7.4 & & \\
\hline & & \multirow{2}{*}{$\begin{array}{l}\text { At least once } \\
\text { per month }\end{array}$} & DNE & 329 & 95.9 & 54 & 91.5 & 1013 & 97.6 & 8.535 & 0.014 \\
\hline & & & EXP & 14 & 4.1 & 5 & 8.5 & 25 & 2.4 & & \\
\hline & & \multirow{2}{*}{$\begin{array}{l}\text { Once per } \\
\text { week }\end{array}$} & DNE & 331 & 96.5 & 57 & 96.6 & 1020 & 98.3 & 4.080 & 0.130 \\
\hline & & & EXP & 12 & 3.5 & 2 & 3.4 & 18 & 1.7 & & \\
\hline & & \multirow{2}{*}{ Daily } & DNE & 338 & 98.5 & 58 & 98.3 & 1031 & 99.3 & 2.200 & 0.333 \\
\hline & & & EXP & 5 & 1.5 & 1 & 1.7 & 7 & 0.7 & & \\
\hline & \multirow{8}{*}{ 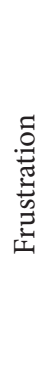 } & \multirow{2}{*}{$\begin{array}{l}\text { Once every } \\
\text { few months }\end{array}$} & DNE & 275 & 80.2 & 38 & 64.4 & 708 & 68.2 & 19.154 & 0.0001 \\
\hline & & & EXP & 68 & 19.8 & 21 & 35.6 & 330 & 31.8 & & \\
\hline & & \multirow{2}{*}{$\begin{array}{l}\text { At least once } \\
\text { per month }\end{array}$} & DNE & 316 & 92.1 & 56 & 93.2 & 866 & 83.4 & 18.829 & 0.0001 \\
\hline & & & EXP & 27 & 7.9 & 4 & 6.8 & 172 & 16.6 & & \\
\hline & & \multirow{2}{*}{$\begin{array}{l}\text { Once per } \\
\text { week }\end{array}$} & DNE & 324 & 94.5 & 58 & 98.3 & 980 & 94.4 & 1.664 & 0.435 \\
\hline & & & EXP & 19 & 5.5 & 1 & 1.7 & 58 & 5.6 & & \\
\hline & & \multirow{2}{*}{ Daily } & DNE & 336 & 98.0 & 58 & 98.3 & 1014 & 97.7 & 0.166 & 0.920 \\
\hline & & & EXP & 7 & 2.0 & 1 & 1.7 & 24 & 2.3 & & \\
\hline
\end{tabular}

Notes: DNE - Did not experience; EXP - Experienced.

Table 3. Frequency and nature of bullying and harassment actions: individual, combat, team sports types (b)

\begin{tabular}{|c|c|c|c|c|c|c|c|c|c|c|}
\hline \multirow{2}{*}{\multicolumn{3}{|c|}{ Actions }} & \multirow{2}{*}{\multicolumn{2}{|c|}{$\begin{array}{c}\text { Individual } \\
\text { sports } N=343 \\
\text { Quantity } \mid \%\end{array}$}} & \multirow{2}{*}{\multicolumn{2}{|c|}{$\begin{array}{l}\text { Combat sports } \\
\qquad \mathrm{N}=59 \\
\text { Quantity } \mid \%\end{array}$}} & \multirow{2}{*}{\multicolumn{2}{|c|}{$\begin{array}{c}\text { Team sports } \\
\mathrm{N}=1038 \\
\text { Quantity } \mid \%\end{array}$}} & \multicolumn{2}{|c|}{$\begin{array}{l}\text { Chi-square } \\
\text { test results }\end{array}$} \\
\hline & & & & & & & & & $x^{2}$ & $\mathrm{P}$ \\
\hline \multirow{4}{*}{ 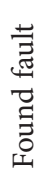 } & \multirow{2}{*}{$\begin{array}{l}\text { Once every } \\
\text { few months }\end{array}$} & DNE & 217 & 63.3 & 39 & 66.1 & 508 & 48.9 & \multirow{2}{*}{25.445} & \multirow{2}{*}{0.0001} \\
\hline & & EXP & 126 & 36.7 & 20 & 33.9 & 530 & 51.1 & & \\
\hline & \multirow{2}{*}{$\begin{array}{l}\text { At least once } \\
\text { per month }\end{array}$} & DNE & 302 & 88.0 & 49 & 83.1 & 846 & 81.5 & \multirow{2}{*}{7.870} & \multirow{2}{*}{0.020} \\
\hline & & EXP & 41 & 12.0 & 10 & 16.9 & 192 & 18.5 & & \\
\hline
\end{tabular}


1534 J. Vveinhardt et al. The dark side of sport: managerial bullying and harassment challenges in different...

End of Table 3

\begin{tabular}{|c|c|c|c|c|c|c|c|c|c|c|}
\hline & \multirow{2}{*}{\multicolumn{2}{|c|}{ Actions }} & \multirow{2}{*}{\multicolumn{2}{|c|}{$\begin{array}{c}\text { Individual } \\
\text { sports } \mathrm{N}=343\end{array}$}} & \multirow{2}{*}{\multicolumn{2}{|c|}{$\begin{array}{c}\text { Combat sports } \\
\mathrm{N}=59 \\
\text { Quantity } \mid \%\end{array}$}} & \multirow{2}{*}{\multicolumn{2}{|c|}{$\begin{array}{c}\text { Team sports } \\
\mathrm{N}=1038 \\
\text { Quantity } \mid \%\end{array}$}} & \multicolumn{2}{|c|}{$\begin{array}{l}\text { Chi-square } \\
\text { test results }\end{array}$} \\
\hline & & & & & & & & & $x^{2}$ & $\mathrm{P}$ \\
\hline & \multirow{2}{*}{$\begin{array}{l}\text { Once per } \\
\text { week }\end{array}$} & DNE & 321 & 93.6 & 49 & 83.1 & 936 & 90.2 & \multirow{2}{*}{7.816} & \multirow{2}{*}{0.020} \\
\hline & & EXP & 22 & 6.4 & 10 & 16.9 & 102 & 9.8 & & \\
\hline & \multirow{2}{*}{ Daily } & DNE & 325 & 94.8 & 52 & 88.1 & 994 & 95.8 & \multirow{2}{*}{7.321} & \multirow{2}{*}{0.026} \\
\hline & & EXP & 18 & 5.2 & 7 & 11.9 & 44 & 4.2 & & \\
\hline \multirow{8}{*}{ 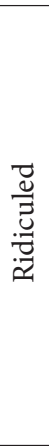 } & \multirow{2}{*}{$\begin{array}{l}\text { Once } \\
\text { every few } \\
\text { months }\end{array}$} & DNE & 250 & 72.9 & 43 & 72.9 & 614 & 59.2 & \multirow{2}{*}{23.443} & \multirow{2}{*}{0.0001} \\
\hline & & EXP & 93 & 27.1 & 16 & 27.1 & 424 & 40.8 & & \\
\hline & \multirow{2}{*}{$\begin{array}{l}\text { At least } \\
\text { once per } \\
\text { month }\end{array}$} & DNE & 312 & 91.0 & 53 & 89.8 & 901 & 86.8 & \multirow{2}{*}{4.413} & \multirow{2}{*}{0.110} \\
\hline & & EXP & 31 & 9.0 & 6 & 10.2 & 137 & 13.2 & & \\
\hline & \multirow{2}{*}{$\begin{array}{l}\text { Once per } \\
\text { week }\end{array}$} & DNE & 325 & 94.8 & 54 & 91.5 & 974 & 93.8 & \multirow{2}{*}{1.024} & \multirow{2}{*}{0.599} \\
\hline & & EXP & 18 & 5.2 & 5 & 8.5 & 64 & 6.2 & & \\
\hline & \multirow{2}{*}{ Daily } & DNE & 329 & 95.9 & 55 & 93.2 & 1017 & 98.0 & \multirow{2}{*}{8.016} & \multirow{2}{*}{0.018} \\
\hline & & EXP & 14 & 4.1 & 4 & 6.8 & 21 & 2.0 & & \\
\hline \multirow{8}{*}{ 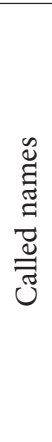 } & \multirow{2}{*}{$\begin{array}{l}\text { Once } \\
\text { every few } \\
\text { months }\end{array}$} & DNE & 260 & 75.8 & 38 & 64.4 & 596 & 57.4 & \multirow{2}{*}{37.154} & \multirow{2}{*}{0.0001} \\
\hline & & EXP & 83 & 24.2 & 21 & 35.6 & 442 & 42.6 & & \\
\hline & \multirow{2}{*}{$\begin{array}{l}\text { At least } \\
\text { once per } \\
\text { month }\end{array}$} & DNE & 300 & 87.5 & 52 & 88.1 & 833 & 80.3 & & \\
\hline & & EXP & 43 & 12.5 & 7 & 11.9 & 205 & 19.7 & 10.646 & 0.005 \\
\hline & Once per & DNE & 315 & 91.8 & 54 & 91.5 & 933 & 89.9 & 1221 & 0542 \\
\hline & week & EXP & 28 & 8.2 & 5 & 8.5 & 105 & 10.1 & 1.221 & 0.543 \\
\hline & . & DNE & 326 & 95.0 & 54 & 91.5 & 987 & 95.1 & 1402 & 0476 \\
\hline & Ddily & EXP & 17 & 5.0 & 5 & 8.5 & 51 & 4.9 & $1.40 \mathrm{~J}$ & 0.470 \\
\hline & Once & DNE & 313 & 91.3 & 50 & 84.7 & 815 & 78.5 & & \\
\hline & $\begin{array}{l}\text { every few } \\
\text { months }\end{array}$ & EXP & 30 & 8.7 & 9 & 15.3 & 223 & 21.5 & 28.459 & 0.0001 \\
\hline & At least once & DNE & 327 & 95.3 & 54 & 91.5 & 961 & 92.6 & 3.352 & 0187 \\
\hline uै & per month & EXP & 16 & 4.7 & 5 & 8.5 & 77 & 7.4 & & \\
\hline 苾 & Once per & DNE & 329 & 95.9 & 53 & 89.8 & 1007 & 97.0 & 8016 & 0012 \\
\hline & week & EXP & 14 & 4.1 & 6 & 10.2 & 31 & 3.0 & 8.816 & 0.012 \\
\hline & $\mathrm{D}_{2} \mathrm{l}_{\mathbf{Y}}$ & DNE & 335 & 97.7 & 54 & 91.5 & 1022 & 98.5 & 12820 & (1) \\
\hline & Dally & EXP & 8 & 2.3 & 5 & 8.5 & 16 & 1.5 & 13.830 & 0.001 \\
\hline
\end{tabular}

Notes: DNE - Did not experience; EXP - Experienced. 
Table 4. Lack of bullying and harassment control/management: individual, combat, team sports types

\begin{tabular}{|c|c|c|c|c|c|c|c|c|c|}
\hline \multirow{2}{*}{\multicolumn{2}{|c|}{$\begin{array}{l}\text { Lack of bullying and } \\
\text { harassment control/ } \\
\text { management }\end{array}$}} & \multicolumn{2}{|c|}{$\begin{array}{l}\text { Individual } \\
\text { sports } \\
(\mathrm{N}=343)\end{array}$} & \multicolumn{2}{|c|}{$\begin{array}{c}\text { Combat sports } \\
\quad(\mathrm{N}=59)\end{array}$} & \multicolumn{2}{|c|}{$\begin{array}{c}\text { Team sports } \\
(\mathrm{N}=1038)\end{array}$} & \multicolumn{2}{|c|}{$\begin{array}{l}\text { ANOVA test } \\
\text { results }\end{array}$} \\
\hline & & Mean & SD & Mean & SD & Mean & SD & $\mathrm{F}$ & $\mathrm{p}$ \\
\hline \multirow{3}{*}{$\begin{array}{l}\text { Affirmation, } \\
\text { intervention } \\
\text { and } \\
\text { prevention of } \\
\text { bullying and } \\
\text { harassment }\end{array}$} & $\begin{array}{l}\text { Seeing the } \\
\text { situation, fact } \\
\text { statement }\end{array}$ & 1.81 & 1.02 & 1.84 & 0.98 & 2.07 & 1.01 & 9.322 & 0.0001 \\
\hline & $\begin{array}{l}\text { Bullying and } \\
\text { harassment } \\
\text { intervention }\end{array}$ & 1.51 & 0.75 & 1.50 & 0.73 & 1.72 & 0.83 & 9.877 & 0.0001 \\
\hline & $\begin{array}{l}\text { Bullying and } \\
\text { harassment } \\
\text { prevention }\end{array}$ & 1.60 & 0.87 & 1.49 & 0.77 & 1.83 & 0.93 & 10.697 & 0.0001 \\
\hline
\end{tabular}

The results unfolded in Table 4 show that bullying and harassment experiences statistically significantly differ, depending on type of sports. The same statistically significant differences were also found in the area of prevention and intervention application. Less importance is attached to the lack of bullying and harassment management among combat and individual sports athletes. Representatives of team sports more often state the absence of management/ control of the phenomena.

Analysing athletes' opinion on prevalence of bullying, it was found that 21.3 per cent acknowledged that bullying and harassment were a major problem in the sport they represented.

From the standpoint of bullying and harassment intervention, it was found that persons working in a team/group (coaches, managers, doctors, other employees of sport club) did not give ear to both sides (12.4\%). That is, the problem arisen is not always thoroughly investigated, which can be the reason for wrong decisions and aggravate the conflict.

A similar problem is also relevant in the area of prevention of both bullying and harassment. A significant share of athletes stated being dissatisfied with the actions of their team/ group, seeking to avoid bullying and harassment among athletes (14.2\%). In other words, this reveals insufficient attention to regulation of interrelationships (there are no clear rules or they are not observed) and to promotion of reporting intolerable behaviour of colleagues.

The athlete makes a decision to report the experienced negative behaviour and seek help, depending on relationships with the social environment. Although no statistically significant differences were found, there are indications showing dependence of behavioural differences on the group of sports. In the instances of bullying and harassment, the situation in teams/ groups is less often reported to trustworthy individuals by representatives of combat and team sports; and more often, by representatives of individual sports (Table 5). On the other hand, the most trusted individuals to whom athletes tell about bullying and harassment experienced in the team/group are those in their immediate environment: parents $(38.8 \%)$, friends who are not related to sport (37\%), the coach (31.9\%). Persons who are less close to athletes but who made up the highest percentage in this group (19.2\%) are the top managers of the institution (sport club or sport school). 
Table 5. Trustworthy persons and actions that have helped to endure bullying and harassment: individual, combat, team sports types

\begin{tabular}{|c|c|c|c|c|c|c|c|c|c|}
\hline \multirow{2}{*}{\multicolumn{2}{|c|}{$\begin{array}{l}\text { Trustworthy persons and } \\
\text { actions that have helped to } \\
\text { endure bullying }\end{array}$}} & \multicolumn{2}{|c|}{$\begin{array}{c}\text { Individual } \\
\text { sports } \\
(\mathrm{N}=343)\end{array}$} & \multicolumn{2}{|c|}{$\begin{array}{l}\text { Combat sports } \\
\qquad(\mathrm{N}=59)\end{array}$} & \multicolumn{2}{|c|}{$\begin{array}{l}\text { Team sports } \\
(\mathrm{N}=1038)\end{array}$} & \multicolumn{2}{|c|}{$\begin{array}{l}\text { ANOVA test } \\
\text { results }\end{array}$} \\
\hline & & Mean & SD & Mean & SD & Mean & SD & $\mathrm{F}$ & $\mathrm{p}$ \\
\hline \multirow{2}{*}{$\begin{array}{l}\text { Trustworthy } \\
\text { persons } \\
\text { from the } \\
\text { victim's } \\
\text { standpoint }\end{array}$} & $\begin{array}{l}\text { Less close } \\
\text { persons and/ } \\
\text { or strangers } \\
\text { who can help }\end{array}$ & 2.27 & 1.24 & 2.01 & 1.15 & 1.90 & 1.01 & 15.340 & 0.0001 \\
\hline & $\begin{array}{l}\text { Persons in } \\
\text { the imme- } \\
\text { diate en- } \\
\text { vironment }\end{array}$ & 3.03 & 1.35 & 2.66 & 1.19 & 2.57 & 1.15 & 18.888 & 0.0001 \\
\hline \multirow{2}{*}{$\begin{array}{l}\text { Actions that } \\
\text { have helped } \\
\text { to endure } \\
\text { bullying }\end{array}$} & $\begin{array}{l}\text { Informing } \\
\text { and } \\
\text { conversations }\end{array}$ & 2.96 & 1.28 & 2.88 & 1.35 & 2.94 & 1.11 & 0.128 & 0.880 \\
\hline & $\begin{array}{l}\text { Preventive } \\
\text { education } \\
\text { and self- } \\
\text { development }\end{array}$ & 2.58 & 1.21 & 2.54 & 1.21 & 2.41 & 1.03 & 3.502 & 0.030 \\
\hline
\end{tabular}

According to athletes, the actions that have helped to endure bullying and harassment (the ones that were performed in general) are as follows: 48.3 per cent indicated that the coach had had a conversation in a team/group; 40.6 per cent noted that information about bullying and harassment had been provided, and 38.2 per cent noted that information on how to report bullying and harassment had been provided. Thus, it can be assumed that informing and conversations are among the actions taken by coaches once they become aware of bullying or harassment in their coached teams/groups. However, they are applied relatively rarely (more than half of respondents did not state such actions). Other answers of respondents also show particularly little attention paid to prevention. That is, only 24.5 per cent of athletes knew that trainings on how to deal with bullying incidents for coaches and other team members had been organised, and only 23.1 per cent of respondents stated that lectures on anti-bullying policy in sport for athletes had been organized.

\section{Discussion}

Our study shows that both prevalence of bullying and harassment and their forms depend on the type of sports, which encourages to look for a specific management approach. Steinfeldt et al. (2012) emphasize that struggling with negative behaviour, anti-bulling policy makers should first of all assess the situation with regard to both the given population and the specific context. For example, within the scope of this study, as many as one fifth of respondents representing organized sport in Lithuania stated the existence of the problem of negative interrelationships. Compared with studies in other countries, this indicator is quite high. 
For example, the study with 1458 male adolescent athletes from 9 different sports and 97 different sport clubs, conducted in Portugal, revealed that about 10 per cent of athletes had identified themselves as victims of bullying (Nery et al., 2019). In addition, it was found that the victims had experienced bullying actions quite rarely and for a short time and mostly they were related to verbal form of bullying. Verbal bullying as the most prevalent in sport is also indicated by Australian Sport Commission investigation (Mattey et al., 2014). Similar results related to verbal form of bullying and relatively low frequency were also found in another study with participants of organized sports, conducted in Portugal (Marracho et al., 2019). In Canada, Mishna et al. (2019), who examined 122 student-age athletes from 24 sports, found that 48 per cent of respondents had stated being bullied. The most common form was relational aggression, which, in respondents' opinion, is considered to be common practice in sport (Kerr et al., 2016). Our study confirms more common forms of verbal violence, but provides a broader picture of the forms of aggression used, showing that actions related to the victim's social isolation and belittling of reputation pose no less risks. Besides, both the forms and frequency of aggression itself vary depending on the type of sports. Team sport is based on interactions between the members of all team, both seeking personal goals and the team goal. This division may have a mitigating effect on interpersonal competition, while in combat and individual sports, interpersonal competition is intensified. This can pertain to the increased incidence of bullying and harassment, noticed in these groups, as a by-product of such competition.

Recent research shows the existence of a still unresolved problem related to assurance of athletes' safety and implies that personal costs of bullying to athletes can be long term and extremely damaging (Mountjoy et al., 2016), which can be viewed as a general problem of managing sports organizations, which is still dealt with difficulty. It is also supported by the culture formed within sports organizations, which together with the lack of formal procedures can influence avoidance of reporting experienced bullying and harassment. For example, it is noted that managers and coaches of sports organizations themselves and often even athletes themselves, being afraid of potential damage to their team or to the reputation of their sports organisation, due to certain agreements or ignorance, deny the existing problem or do not acknowledge it (Mountjoy et al., 2016). If the organization does not pay enough attention to prevention of negative relationships, this can form a misconception of the organizational values. Therefore, values diminishing involvement in bullying behaviour should be supported by all members, athletes, coaches, administrators, and managers of the organization's community without exception (Waldron, 2012), and especially by those whom athletes treat as authorities. The results of our study demonstrate that equating coaches with the immediate environment (as family members and friends) can provide an additional stimulus for bullying and harassment intervention. Therefore, there is a need for greater involvement of this segment of the organization, which is currently extremely low. A fairly high level of trust in coaches will also influence athletes' values and formation of behavioural norms, which will provide additional impetus for prosocial behaviour. Such approach agrees with the study conducted by Steinfeldt et al. (2012), emphasizing that parents, brothers and team leaders may not only influence the development of the male role but that these men can positively affect adolescent football players' bullying and behaviour. 
However, the problem of coaches' competence remains relevant. Although nearly onethird of respondents in our study stated that they would address the coach, coaches often lack competence in interpersonal relationships, while work ethics prevailing in the sports organisation compels them not to give sufficient attention to bullying and harassment incidents (Kerr et al., 2016). This explains the lack of attention to the rules governing interrelationships. It may be that coaches are more confident in their authority and competence than in official measures. On the other hand, the overall scope of the phenomenon may signal that disregard of formal rules is a more general consequence of the system formed by leadership traditions characteristic to this population, which have developed over time. While the greatest weight of operative conflict resolution falls on coaches, institutionalisation of relationship norms and implementation of prevention programs is the organisation's prerogative. Therefore, the organization also bears responsibility for coaches' competencies, both organizing their development and encouraging coaches to do this themselves. For example, a significant share of athletes knew nothing about bullying and harassment prevention, which points to shortcomings of the organizational system. Besides, the fact that coaches are far from giving ear to all sides of the conflict is likely to signal the lack of competence, which, if compensated, allows to obtain abilities to respond to the situation appropriately (Shannon, 2013). All of it leads to the assumption that at the organizational level, proper supervision of interpersonal relationships is not ensured and that the organizational culture favourable for elimination of bullying and harassment is not created (e.g., Mountjoy et al., 2016). In other words, education should become the basis of any safe sports strategy, since prevention actually begins with awareness of bullying and harassment, their nature and health consequences (Mountjoy et al., 2016). Nevertheless, the most efficient way to prevent bullying and harassment in a separate sports organization should be a systemic multilateral approach (Fisher \& Dzikus, 2017). This should not only encompass education of all who are present in the sports environment but should be linked to the formation of the organization's culture that is unfavourable to bullying and harassment, considering the cultural context, while "safe sport" should become the element of good governance in sport, defended in a top-down way in every sports organization (Van Rheenen, 2013).

\section{Conclusions}

This study broadens knowledge of prevalence of bullying and harassment in organized sports, depending on the type of sports, and grounds the idea that the accents of sport organization's management in applying preventive and intervention measures should differ. This means that the context of the individual types not only influences the level of interpersonal competitiveness but also determines behaviour that is conflicting to a greater or lesser degree in the relationships with other athletes. On the one hand, it is evident that the athlete's close relationship with the coach offers untapped opportunities that have to be considered by both coaches themselves and the organisations' top managers. On the other hand, organising intervention and prevention of bullying and harassment, it is important to note that violence manifests itself not only verbally but also in physical actions. Not only negative communication but also the victim's isolation and belittling of reputation receive insufficient attention 
because they are more difficult to spot and are regulated by rules but cause no less painful experiences. Isolation and degrading reputation are a deceptive form of violence against victims, manifesting itself as unfair competition. This way, the focus solely on evident bullying and harassment in preventive measures and intervention programs will give only a partial effect and may promote a shift towards less noticeable dishonest behaviour.

Limitations and further research: Despite the new insights into bullying and harassment in sports, this article presents only the part of the research results, that reveals the prevalence of bullying and harassment in organized sport in Lithuanian and intervention actions at the organizational level based solely on the opinion of athletes. All results are limited to such criteria as country, age, sport types and sport level. Athletes under the age of 16 or over the age of 29 were excluded from the study, the study did not cover absolutely all sports in Lithuania and the level of sport participation of athletes - amateur sport.

In the future, it would make sense to conduct surveys of sports coaches and top managers of sports organizations, which will allow to compare the established prevalence of bullying and harassment and disclosed intervention actions or management activities. Also extend the research to a broader intercultural context by conducting research in culturally close countries and comparing research results.

\section{Funding}

This study is part of a project which has received funding from European Social Fund (project No 09.3.3-LMT-K-712-01-0190) under grant agreement with the Research Council of Lithuania (LMTLT).

\section{Author contributions}

Conceptualization, J.V. and V.B.F.; methodology, J.V. and V.B.F.; software, J.V.; formal analysis, J.V. and V.B.F.; investigation, R.A. and V.B.F.; resources, J.V. and V.B.F.; data curation, J.V.; visualization, J.V. and V.B.F.; writing - original draft preparation, J.V. and V.B.F.; writing review and editing, J.V. and V.B.F.

\section{Disclosure statement}

The authors declare no conflict of interest.

\section{References}

Bachand, C. R. (2017). Bullying in Sports: the definition depends on who you ask. The Sport Journal, 9, $1-14$.

Buss, A. H., \& Durkee, A. (1957). An inventory for assessing different kinds of hostility. Journal of Consulting Psychology, 21(4), 343-349. https://doi.org/10.1037/h0046900

Cosgrove, H. E., \& Nickerson, A. B. (2017). Anti-bullying/harassment legislation and educator perceptions of severity, effectiveness, and school climate: A cross-sectional analysis. Educational Policy, 31(4), 518-545. https://doi.org/10.1177/0895904815604217 
Crawshaw, L. (2009). Workplace bullying? Mobbing? Harassment? Distraction by a thousand definitions. Consulting Psychology Journal: Practice and Research, 61(3), 263-267. https://doi.org/10.1037/a0016590

Donohue, B., Chow, G. M., Pitts, M., Loughran, T., Schubert, K. N., Gavrilova, Y., \& Allen, D. N. (2015). Piloting a family-supported approach to concurrently optimize mental health and sport performance in athletes. Clinical Case Studies, 14(3), 159-177. https://doi.org/10.1177/1534650114548311

Espelage, D. L., \& Holt, M. (2001). Bullying and victimization during early adolescence: Peer influences and psychosocial correlates. Journal of Emotional Abuse, 2(2-3), 123-142. https://doi.org/10.1300/J135v02n02_08

Evans, B., Adler, A., MacDonald, D., \& Côté, J. (2016). Bullying victimization and perpetration among adolescent sport teammates. Pediatric Exercise Science, 28(2), 296-303. https://doi.org/10.1123/pes.2015-0088

Fisher, L. A., \& Dzikus, L. (2017). Bullying in Sport and Performance Psychology. In E. Acevedo (Eds.), Oxford research encyclopedia of psychology (pp. 1-4). Oxford University Press. https://doi.org/10.1093/acrefore/9780190236557.013.169

Gillen, P., Sinclair, M., \& Kernohan, G. (2004). A concept analysis of bullying in midwifery. EvidenceBased Midwifery, 2(2), 46-52.

Hellström, L., Persson, L., \& Hagquist, C. (2015). Understanding and defining bullying-adolescents' own views. Archives of Public Health, 73(1), 4. https://doi.org/10.1186/2049-3258-73-4

Holt, N. L., Neely, K. C., Slater, L. G., Camiré, M., Côté, J., Fraser-Thomas, J., MacDonald, D., Strachan, L., \& Tamminen, K. A. (2017). A grounded theory of positive youth development through sport based on results from a qualitative meta-study. International Review of Sport and Exercise Psychology, 10(1), 1-49. https://doi.org/10.1080/1750984X.2016.1180704

Jewett, R., Kerr, G., MacPherson, E., \& Stirling, A. (2019). Experiences of bullying victimisation in female interuniversity athletes. International Journal of Sport and Exercise Psychology. https://doi.org/10.1080/1612197X.2019.1611902

Kavussanu, M., \& Boardley, I. D. (2009). The prosocial and antisocial behavior in sport scale. Journal of Sport and Exercise Psychology, 31(1), 97-117. https://doi.org/10.1123/jsep.31.1.97

Kerr, G., Jewett, R., MacPherson, E., \& Stirling, A. (2016). Student-athletes' experiences of bullying on intercollegiate teams. Journal for the Study of Sports and Athletes in Education, 10(2), 132-149. https://doi.org/10.1080/19357397.2016.1218648

Kerr, Z. Y., Roos, K. G., Schmidt, J. D., \& Marshall, S. W. (2013). Prevention and management of physical and social environment risk factors for sports-related injuries. American Journal of Lifestyle Medicine, 7(2), 138-153. https://doi.org/10.1177/1559827612450683

Lund, E. M., \& Ross, S. W. (2017). Bullying perpetration, victimization, and demographic differences in college students: A review of the literature. Trauma, Violence, \& Abuse, 18(3), 348-360. https://doi.org/10.1177/1524838015620818

Marracho, P., Pereira, A., Nery, M., \& Coelho, E. (2019). Bullying in school sports vs federated sports: Exploratory study in the interior Northern region of Portugal. Motricidade, 15(1), 210-210.

Mattey, E., McCloughan, L. J., \& Hanrahan, S. J. (2014). Anti-vilification programs in adolescent sport. Journal of Sport Psychology in Action, 5(3), 135-146. https://doi.org/10.1080/21520704.2014.925528

Maxwell, J. P., \& Moores, E. (2007). The development of a short scale measuring aggressiveness and anger in competitive atletes. Psychology of Sport and Exercise, 8(2), 179-193. https://doi.org/10.1016/j.psychsport.2006.03.002

McMullen, J. G. (2014). Addressing abusive conduct in youth sports. Marquette Sports Law Review, 25(1), 181-205.

Merkel, D. L. (2013). Youth sport: Positive and negative impact on young athletes. Open Access Journal of Sports Medicine, 4, 151-160. https://doi.org/10.2147/OAJSM.S33556 
Menesini, E., \& Salmivalli, C. (2017). Bullying in schools: the state of knowledge and effective interventions. Psychology, Health \& Medicine, 22(sup1), 240-253.

https://doi.org/10.1080/13548506.2017.1279740

Mishna, F., Kerr, G., McInroy, L. B., \& MacPherson, E. (2019). Student athletes' experiences of bullying in intercollegiate sport. Journal for the Study of Sports and Athletes in Education, 13(1), 53-73. https://doi.org/10.1080/19357397.2019.1581512

Moore, S. E., Norman, R. E., Suetani, S., Thomas, H. J., Sly, P. D., \& Scott, J. G. (2017). Consequences of bullying victimization in childhood and adolescence: A systematic review and meta-analysis. World Journal of Psychiatry, 7(1), 60-76. https://doi.org/10.5498/wjp.v7.i1.60

Mountjoy, M., Brackenridge, C., Arrington, M., Blauwet, C., Carska-Sheppard, A., Fasting, K., Kirby, S., Leahy, T., Marks, S., Martin, K., Starr, K., Tiivas, A., \& Budgett, R. (2016). International Olympic Committee consensus statement: Harassment and abuse (non-accidental violence) in sport. British Journal of Sports Medicine, 50(17), 1019-1029. https://doi.org/10.1136/bjsports-2016-096121

Nery, M., Neto, C., Rosado, A., \& Smith, P. K. (2019). Bullying in youth sport training: A nationwide exploratory and descriptive research in Portugal. European Journal of Developmental Psychology, 16(4), 447-463. https://doi.org/10.1080/17405629.2018.1447459

Notelaers, G., \& Van der Heijden, B. I. J. M. (2019). Construct validity in workplace bullying and harassment research. In P. D'Cruz, E. Noronha, G. Notelaers, \& C. Rayner (Eds.), Concepts, approaches and methods. Handbooks of workplace bullying, emotional abuse and harassment (pp. 1-56). Springer, Singapore. https://doi.org/10.1007/978-981-10-5334-4_11-1

Patchin, J. W., \& Hinduja, S. (2015). Measuring cyberbullying: Implications for research. Aggression and Violent Behavior, 23, 69-74. https://doi.org/10.1016/j.avb.2015.05.013

Peltola, M., \& Kivijärvi, A. (2017). Sports and structured leisure as sites of victimization for children and young people in Finland: Looking at the significance of gender and ethnicity. International Review for the Sociology of Sport, 52(8), 955-971. https://doi.org/10.1177/1012690216636607

Pitts, B. G., Fielding, L. W., \& Miller, L. K. (1994). Industry segmentation theory and the sport industry: Developing a sport industry segment model. Sport Marketing Quarterly, 3(1), 15-24.

Roberts, V., Sojo, V., \& Grant, F. (2019). Organisational factors and non-accidental violence in sport: A systematic review. Sport Management Review.

https://doi.org/10.1016/j.smr.2019.03.001

Shannon, C. S. (2013). Bullying in recreation and sport settings: Exploring risk factors, prevention efforts, and intervention strategies. Journal of Park and Recreation Administration, 31(1), 15-33.

Smith, P. K. (2016). Bullying: Definition, types, causes, consequences and intervention. Social and Personality Psychology Compass, 10(9), 519-532. https://doi.org/10.1111/spc3.12266

Smith, P. K., del Barrio, C., \& Tokunaga, R. (2013). Definitions of bullying and cyberbullying: How useful are the terms? In S. Bauman, J, Walker \& D. Cross (Eds.), Principles of cyberbullying research: Definition, methods, and measures (pp. 64-86). Routledge.

Stefaniuk, L., \& Bridel, W. (2018). Anti-bullying policies in Canadian Sport: An absent presence. Journal of Park \& Recreation Administration, 36(2). https://doi.org/10.18666/JPRA-2018-V36-I2-8439

Steinfeldt, J. A., Vaughan, E. L., LaFollette, J. R., \& Steinfeldt, M. C. (2012). Bullying among adolescent football players: Role of masculinity and moral atmosphere. Psychology of Men \& Masculinity, 13(4), 340-353. https://doi.org/10.1037/a0026645

Stirling, A. E., Bridges, E. J., Cruz, E. L., \& Mountjoy, M. L. (2011). Canadian Academy of Sport and Exercise Medicine position paper: Abuse, harassment, and bullying in sport. Clinical Journal of Sport Medicine, 21(5), 385-391. https://doi.org/10.1097/JSM.0b013e31820f9248

Stirling, A. E., \& Kerr, G. A. (2016). Athlete maltreatment. In R. J. Schinke, K. R. McGannon, \& B. Smith (Eds.), Routledge international handbook of sport psychology (pp. 214-224). Routledge. 
1542 J. Vveinhardt et al. The dark side of sport: managerial bullying and harassment challenges in different...

Škorić, S. (2018). Characteristics of sport managers and challenges facing sport organisations. In 9th International Conference "An enterprise odyssey: Managing change to achieve quality development" (pp. 497-503). University of Zagreb, Faculty of Economics \& Business.

Twemlow, S. W., \& Sacco, F. C. (2013). Bullying is everywhere: Ten universal truths about bullying as a social process in schools \& communities. Psychoanalytic Inquiry, 33(2), 73-89. https://doi.org/10.1080/07351690.2013.759484

Van Rheenen, D. (2013). Exploitation in college sports: Race, revenue, and educational reward. International Review for the Sociology of Sport, 48(5), 550-571. https://doi.org/10.1177/1012690212450218

Vveinhardt, J. (2012). Identification of the reliability of methodological characteristics of quality in the diagnostic instrument for mobbing as discrimination in employee relations on purpose to improve the climate in Lithuanian organisations. Transformations in Business \& Economics, 11(2), 218-232.

Vveinhardt, J., \& Andriukaitiene, R. (2017, October 5-7). Bullying and single cases of harassment in women's basketball teams. In International Scientific-Practical Symposium. In collaboration with the European Group for Research into Elderly and Physical Activity "Climbing the Ladder of Life, Active and Fit" (pp. 35-36). Kaunas, Lithuania.

Vveinhardt, J., \& Fominiene, V. B. (2020a). Gender and age variables of bullying in organized sport: Is bullying "grown out of"? Journal of Human Sport and Exercise, 15(4), in press. https://doi.org/10.14198/jhse.2020.154.03

Vveinhardt, J., \& Fominiene, V. B. (2020b). Prevalence of bullying and harassment in youth sport: the case of different types of sport and participant roles. Journal of Human Sport and Exercise (in press).

Vveinhardt, J., Fominiene, V. B., \& Andriukaitiene, R. (2019). “Omerta” in organized sport: Bullying and harassment as determinants of threats of social sustainability at the individual level. Sustainability, 11(9), 2474. https://doi.org/10.3390/su11092474

Vveinhardt, J., Komskiene, D., \& Romero, Z. (2017). Bullying and harassment prevention in youth basketball teams. Transformations in Business \& Economics, 16(1), 232-241.

Vveinhardt, J., \& Streimikiene, D. (2015). The questionnaire for diagnosing mobbing in employees' relationships. Economic Research-Ekonomska Istraživanja, 28(1), 441-466. https://doi.org/10.1080/1331677X.2015.1075415

Vveinhardt, J., Fominiene, V. B., \& Streimikiene, D. (2020). A new tool contributing to the management of bullying and harassment in higher education institutions. Polish Journal of Management Studies, 21(2), 446-461. https://doi.org/10.17512/pjms.2020.21.2.31

Waldron, J. J. (2012). A social norms approach to hazing prevention workshops. Journal of Sport Psychology in Action, 3(1), 12-20. https://doi.org/10.1080/21520704.2011.639854

Yildiz, S. (2015). The relationship between bullying and burnout: An empirical investigation of Turkish professional football players. Sport, Business and Management, 5(1), 6-20.

https://doi.org/10.1108/SBM-09-2012-0034 